\title{
Antiretroviral Toxicity and Oxidative Stress
}

\author{
${ }^{1}$ Adikwu Elias, ${ }^{1}$ Brambaifa Nelson, ${ }^{2}$ Deo Oputiri and ${ }^{2}$ Oru-Bo Precious Geoffrey \\ ${ }^{1}$ Department of Pharmacology, Faculty of Basic Medical Sciences, \\ College of Health Sciences, University of Port Harcourt, Choba, Rivers State, Nigeria \\ ${ }^{2}$ Department of Pharm Tech, College of Health Technology, Otuogidi, Ogbia, L. G. A., Bayelsa State, Nigeria
}

Received 2013-04-23, Revised 2013-10-14; Accepted 2013-11-20

\begin{abstract}
Antiretroviral drugs are used for the treatment of human immunodeficiency virus, they are used as combination regimens to achieve the highest possible benefit, tolerability, compliance and to diminish the risk of resistance development. Reports from preclinical and clinical studies have linked antiretrovirals with some toxicological effects which could be associated with redox imbalance (oxidative stress). This stimulated us to review relevant literature on the relationship between antiretroviral induced toxicological effects and redox imbalance. Available literature on antiretroviral associated toxicological effects and oxidative stress were comprehensively reviewed. Literature showed that antiretrovirals are associated with toxicological effects which includes hepatotoxicity, cardiotoxicity, hematotoxicity and nephrotoxicity. Reports in animal studies also showed that these toxicological effects could be associated with oxidative stress through the generation of oxidative radicals, depletion of antioxidants and antioxidant enzymes leading to mitochondria damage in the heart, kidney, liver brain and other organs. In humans, studies also showed that antiretrovirals are associated with lipid peroxidation, depletion of antoxidants and antioxidant enzymes which are elements of oxidative stress. Furthermore it was observed that supplementations with some antioxidants mitigated antiretroviral induced oxidative stress, mitochondria damage and toxicological effects. Antiretroviral drugs are associated with toxicological effects which may involve redox imbalance, but more studies are required to correlate antiretroviral toxicities with oxidative stress.
\end{abstract}

Keywords: Antiretroviral, Toxicity, Oxidative Stress, Oxidative Radicals, Antioxidants

\section{INTRODUCTION}

Acquired Immune Deficiency Syndrome (AIDS) was identified as a disease in 1981, Human Immunodeficiency Virus (HIV) was isolated as the putative cause of the disease (Barre-Sinoussi et al., 1983; Popovic et al., 1983). This discovery created room for the search for chemical agents that could inhibit infectivity and replication of human immunodeficiency virus. This led to the discovery of the first antiretroviral chemical agent (zidovudine) which was introduced for clinical use in 1987 (Mitsuya et al., 1985). In 2008, 25 years after the Human Immunodeficiency Virus (HIV) was discovered as the cause of Acquired Immune Deficiency
Syndrome (AIDS), exactly 25 antiretroviral compounds have been clinically approved for the management of HIV/AIDS. Currently these compounds fall into six classes: Nucleoside Reverse Transcriptase Inhibitors (NRTIs) Nucleotide Reverse Transcriptase Inhibitors (NtRTIs) Non-Nucleoside Reverse Transcriptase Inhibitors (NNRTIs), Protease Inhibitors (PIs), Fusion Inhibitors (FIs) and Co-Receptor Inhibitors (CRIs) and Integrase Inhibitors (INIs). These compounds are used as combination regimens to achieve the highest possible benefit, tolerability, compliance and to diminish the risk of resistance development (De Clercq, 2009). Currently recommended initial regimens use combinations of two Nucleoside Reverse Transcriptase Inhibitors (NRTIs) and either a Non-

Corresponding Author: Adikwu Elias, Department of Pharmacology, Faculty of Basic Medical Sciences,

College of Health Sciences, University of Port Harcourt, Choba, Rivers State, Nigeria

Tel: $+2347068568868,+2348128209442$ 
Nucleoside Reverse Transcriptase Inhibitor (NNRTI), a ritonavir-boosted Protease Inhibitor or an Integrase Inhibitor (INSTI) (Reust, 2011). These combinations are used to achieve clinical benefits which are part of the primary goals of HIV treatment, which include suppressing the viral load, reducing morbidity, maximizing survival, improving quality of life, restoring and maintaining immunological function and preventing further disease transmission (Hughes et al., 2011). These clinical benefits however are not without toxicological effects associated with highly active antiretroviral therapy.Toxicological effects have been one of the most important limiting factors militating against the success of Highly Active Antiretroviral Therapy (HAART). This may result in decrease adherence to treatment which consequently may lead to virological failure, immunological failure, clinical failure, poor prognosis, switching of regimens, therapy discontinuation and even death (Domingo and Lozano, 2011; Bera et al., 2012). Recent studies have shown that antiretroviral drugs induce oxidative stress via generation of oxidative radicals which may be associated with their toxicological effects (Valle et al., 2013). This literary work evaluates antiretroviral toxicity and its correlation with oxidative stress in humans and animals.

\section{TOXICOLOGICAL EFFECTS OF ANTIRETROVIRAL DRUGS}

\subsection{Toxicological Effects of Nucleoside Reverse Transcriptase Inhibitors (NRTIs)}

Drugs in this class includes zidovudine, didanosine, lamivudine, zalcitabine, emtricitabine and stavudine. They are used widely in combination therapy with non nucleoside reverse transcriptase inhibitors and Protease Inhibitors (PIs) in HIV therapy (Van Huyen et al., 2003). NRTIs mediate their activity by inhibiting the viral reverse transcriptase enzyme, thereby preventing the reverse transcription of viral RNA to DNA within the host cell. These agents inhibit viral replication in early stages of viral life cycle. All NRTIs have to be phosphorylated intracellularly, successively to the 5'mono-, 5'-di- and 5'-triphosphate, before they can interact with the HIV Reverse Transcriptase (RT) as an alternate substrate/competitive inhibitors. In this form they compete with any of the natural substrates (dTTP, dCTP, dATP or dGTP) for the HIV RT reaction (Ravichandran et al., 2008; Valle et al., 2013).

Long-term treatment with NRTIs give rise to a broad spectrum of tissue toxicities, most members in this class are known to be associated with wide variety of tissue damage including hematologic disorders, myopathy, cardiotoxicity, peripheral neuropathies and hepatotoxicity. Hepatotoxicity is said to be common with zidovudine, didanosine and zalcitabine (Glesby, 2002). Human and animal studies have shown that zidovudine is associated with hematologic toxicity like neutropenia, macrocytosis and thrombocytopenia (Hassan et al., 2009; Kaferle and Strzoda, 2009). Stavudine is associated with toxicological effect on lipid profile which could be mitigated by recombinant growth hormone or metformin (Aberg et al., 2009). Abacavir is known for its ability to induce hypersensitivity reaction, stevens Johson syndrome and epidermal necrolysis (Suneeta et al., 2008). Pancreatitis, lactic acidosis and peripheral neuropathy are the toxic crimes affiliated with didanosine use (Guo and Fung, 2004). These heterogeneous toxicological effects of NRTIs are said to be related to defective mitochondrial DNA replication secondary to the NRTI-induced deleterious inhibition of the mitochondrial DNA polymerase gamma (Gerschenson and Brinkman, 2004; Saitoh et al., 2007).

\subsection{Toxicological Effects of Nucleotide Reverse Transcriptase Inhibitors (Nt RTIs)}

Tenofovir was the first (2001) NtRTI approved by the US Food and Drug Administration (FDA) for the treatment of HIV infection. Tenofovir was also approved for the treatment of chronic hepatitis B in adults in 2008. The NtRTIs belong to the Acyclic Nucleoside Phosphonates (ANPs), two ANPs are currently in the market: adefovir (PMEA) and tenofovir [(R)-PMPA]. Like the NRTIs, the NtRTIs eventually act as obligatory chain terminators in competition with dATP, but the NtRTIs need only two phosphorylations to be converted to their active (diphosphate) form, since they are already phosphorylated in their parental forms.They are incorporated into DNA via the phosphonate group which makes their excision by exonucleases more difficult than if they were incorporated via the readily cleavable phosphate group (Ravichandran et al., 2008; Valle et al., 2013). Tenofovir is now a widely used component of antiretroviral regimens for the treatment of naive and experienced patients on the basis of its efficacy and tolerability in clinical trials. US HIV treatment guidelines considered tenofovir as part of all preferred regimens for antiretroviral-naive adults and adolescents (Gallant and Deresinski, 2003). Dispite it clinical success in the management of HIV/AIDS, studies in humans and animals have shown that tenofovir could be nephrotoxic (Young et al., 2009). It is said to be associated with bone demineralization (osteoporosis) especially in young children which could be mitigate by bisphosphonates, calcium and vitamin D (Huang et al., 2009). 


\subsection{Toxicological Effects of Non-Nucleoside Reverse Transcriptase Inhibitors (NNRTIs)}

Non-nucleoside Reverse Transcriptase Inhibitors (NNRTIs) constitute a class of medications that have contributed significantly to the management of Human Immunodeficiency Virus (HIV) infection. Members in this class includes efavirenz, nevirapine, etravirine and the newest agent, rilpivirine, which has recently received FDA approval (Schafer et al., 2011). Unlike NRTIs, they NNRTIs do not require cellular activation to inhibit HIV-1RT. They don't require incorporation into nascent viral DNA. They are noncompetitive inhibitors and bind into a hydrophobic "pocket" in the p66 subunit of HIV-1 RT located close to (but distinct from) the NRTI binding site. NNRTI binding distorts the nearby RT polymerase active site, thus affecting the chemical step of polymerization (De Clercq, 2009). NNRTIs have contributed tremendously to the fight against HIV/AIDS but with reservations on some of their toxicites. Hepatotoxicity is one of the toxicological crimes associated with nevirapine (Rivero et al., 2007). Nevirapine hepatotoxicity is characterised by elevation of transaminases (AST and ALT) levels and hepatocytes necrosis (Elias and Brambaifa, 2013; Elias et al., 2013). Efavirenz is associated with neuropsychiatric effects i.e central nervous system disturbances such as emotional instability, insomnia, hallucinations, impaired concentration and abnormal dreams (Jena et al., 2009).

Etravirine which is one of the newest of this group is associated with gastrointestinal disorders (diarrhea) and rash (Madruga et al., 2007; Katlama et al., 2009; Elsayed and Caldwell, 2010). Rilpivirine is the newest of this class that received FDA approval in 2011 for use in combination with other antiretrovirals for the treatment of HIV-1 infection in naive adults. Reports have shown that rilpivirine may impair psychological function (James et al., 2012).

\subsection{Toxicological Effects of Protease Inhibitors}

The introduction of Protease Inhibitors (PIs) in 1996 have significantly reduced morbidity and mortality due to HIV infection. Since the 1990s, multiple PIs have been approved, with several boosted PIs containing regimens recognized as first-line regimens in antiretroviral therapy (Pallela et al., 1998). PIs mediate their antiviral activity by binding protease enzyme and preventing the cleavage of the gag and gag/pol polyproteins into structural functional proteins and enzymes thereby preventing the formation of new viral particles (Wensing et al., 2010). Saquinavir is the first PI to gain approval from the US Food and Drug Administration (FDA) in 1995, after demonstrating ability to reduce HIV-RNA and increase CD4 cell counts in patients infected with HIV. Ritonavir, indinavir and nelfinavir were later licensed by the FDA (Hughes et al., 2011). Amprenavir, fosamprenavir, atazanavir, darunavir and tipranavir were later introduced clinically. PIs are well tolerated but not without toxicological effects like, hepatotoxicity, nephrotoxicity and cardiotoxicity (Hughes et al., 2011). Metabolic abnormalities associated with the toxic effects of PIs on lipid profile are most commonly associated with lopinavir/ritonavir, fosamprenavir/ritonavir with respect to other PIs (Hill et al., 2009; Tebas, 2008; Noor, 2007). One of the reported toxicological effect of PIs is the impairement in glucose metabolism potentiating hyperglycemia and increase incidence of diabetes mellitus. Indinavir, ritonavir and lopinavir/ritonavir have all shown early changes in glucose metabolism and insulin resistance (Hill et al., 2009; Tebas, 2008). Most PIs have demonstrated relative equivalence with respect to causing hepatotoxicity particularly when boosted with ritonavir (Eron et al., 2010). Cardiotoxicity which could be supported by reports on cardiac conduction abnormalities and myocardial infarction have been loosely associated with the use of lopinavir, nelfinavir, ritonavir, saquinavir and indinavir (Hughes et al., 2011; Lundgren et al., 2009).

\subsection{Toxicological Effects of Entry, Fusion and Integrase Inhibitors}

Efuvirtide is currently the only clinically use fusion inhibitor that is approved. Entry and fusion inhibitors block receptors thereby preventing the virus from entering cells. Enfuvirtide is used primarily in the treatment of experienced patients with limited therapeutic options. However, it is a painful subcutaneous injection and is not commonly used. It could be associated with hematotoxicity (eosinophilic and neutropenia) which may increase the risk of bacterial pneumonia.

Integrase strand transfer inhibitors prevent viral DNA from integrating into host DNA by inhibiting the integrase enzyme involved in strand transfer. Raltegravir is the first drug in this class to be approved for use in both treatment of naive and treatment of experienced patients and showed a remarkable lack of relevant adverse effects (Emery and Winston, 2009; Havlir, 2008). Resistance to raltegravir develops easily, which may limit its long-term effectiveness. Myopathy and rhabdomyolysis have been reported as toxicological effects associated with raltegravir. Also raltegravir is associated with fewer 
central nervous system and neuropsychiatric toxicological effects (Lennox et al., 2009).

Maraviroc, which is an entry inhibitor, is the only available CCR5 antagonist. It is approved for use in the treatment of naive and experienced patients. Maraviroc works only on R5 cells; therefore, an R5 tropism test should be performed before initiating therapy with maraviroc. Maraviroc is reported to be associated with bronchitis, nasopharyngitis and esophageal candidiasis. There is evidence of maraviroc induced hepatotoxicity (Meanwell and Kadow, 2007). Trials with miraviroc have shown that it has a favorable safety profile but it could be associated with non-significant changes in total cholesterol, low density lipoprotein, high density lipoprotein and triglycerides (Cooper et al., 2010).

\section{OXIDATIVE STRESS}

Oxidative stress is a terminology usually used to describe the damage caused by Reactive Oxygen Species (ROS) to tissue or organs. Oxidative stress can be defined as an imbalance between the antioxidants and pro-oxidant systems with the shift towards the prooxidant system. Oxidative stress could also be defined as the modification and accumulation of biological molecules altered by various kinds of Reactive Oxygen Species (ROS) and Reactive Nitrogen Species (RNS). ROS and RNS affect gene transcription and cell growth/proliferation and they have been considered as intercellular signal molecules. ROS and RNS are highly reactive, toxic oxygen or nitrogen moieties which includes hydroxyl radical, peroxyl radical, superoxide anion, hydrogen peroxide, nitric oxide and peroxynitrite (Pocernich et al., 2005). The pro-oxidative mechanism includes anion superoxide, hydrogen peroxide and the singlet oxygen. The enzymatic antioxidative system includes superoxide dismutase, catalase and glutathione peroxidase. The non-enzymatic include bilirubin, ceruloplasmin, sexual hormones, melatonin, coenzyme Q and uric acid. In addition to that, other antioxidants are ingested through diet such as ascorbic acid (vitamin c), tocoferol, carotene and flavonoids. The enzymatic antioxidative system and non-enzymatic system prevent the activities of these pro- oxidants (Schneider and De Oliveira, 2004).

Collectively, ROS can lead to oxidation of proteins, DNA, peroxidation of lipids and ultimately cell death. Alterations in proteins by ROS can lead to aggregation, changes in secondary and tertiary structure, susceptibility to proteolysis, fragmentation and loss of function. Oxidative stress is implicated in the pathogenesis of many diseases such as alzheimer disease, diabetes, hypercholesterolemia, hepatorenal syndrome and progression of diseases such as hypertension, atherosclerosis, cardiac hypertrophy and myocardial infarction (Abrescia and Golino, 2005; Terry et al., 2006). Could ROS be associated with antiretroviral induced toxicological effects? Substantial amount of evidence revealed role of OS as a causative factor in the progression of many diseases and drug induce toxicities (Kashou and Agarwal, 2011).

\subsection{Antiretroviral Induced Oxidative Stress in Animals}

Some experimenal studies involving animals have tried to establish a relationship between antiretroviral toxicity and oxidative stress. One of these studies is work of Ferraresi and colleagues who exposed U937 and CEM cell lines to zidovudine, stavudine and didanosine and reported apoptosis and increased intracellular hydrogen peroxide but not superoxide anions. The addition of Acetyl-L-Carnitine (ALC) was able to prevent the prooxidant effect of the these drugs. Supplementation with ALC, deficient in certain cohorts of HIV-infected individuals, especially on high active antiretroviral therapy, has been associated with favourable effects (Ferraresi et al., 2006). Didanosine was reported to induce oxidative stress in the brain mitochondria of animal via increase in protein carbonyl which was mitigated by the administration of brain accessible antioxidants and glutathione mimetic (Lauderback et al., 2003; Sultana et al., 2004; Opii et al., 2007).

The viability of hCMEC/D3 cells (in vitro model of $\mathrm{BBB}$ ) exposed to zidovudine and indinavir decreased significantly after $72 \mathrm{hrs}$ treatment, in a dose-dependent manner. This was atributed to oxidative stress due to altered levels of oxidative stress markers, such as glutathione and malondialdehyde. Pretreatment with the thiol antioxidant $\mathrm{N}$-acetylcysteine reversed some of the pro-oxidant effects of zidovudine and indinavir (Manda et al., 2011). Zidovudine and indinavir are known to be associated associate with some cardiovascular effects eg atherosclerosis which could be attributed to oxidative stress (Jiang et al., 2007; 2009). Efavirenz which is known to be associated with central nervous system disorders was reported to induce oxidative stress in the intracranial visual relay centers of adult wistar rats (Adjene et al., 2011). Efevirenz has also shown evidence for the involvement of mitochondrial dysfunction and oxidative stress in its cellular toxicity in animals (Apostolova et al., 2010). HIV transgenic mice exposed to tenofovir showed ultrastructural 
mitochondrial abnormalities and decreased proximal tubular DNA, but no optical microscopical changes were obsrved. This mitochondria damage is reported to be associated with tenofovir induced nephrotoxicity in humans and animals (Kohler et al., 2009). Significant increase in protein carbonyl content, decrease in glutathione and protein thiol, was observed in the kidney of tenofovir treated rats. Also decreases in the activities of antioxidant enzymes such as superoxide dismutase, glutathione peroxidase, glutathione $\mathrm{S}$ transferase and glutathione reductase and a massive increase in myeloperoxidase activity were observed. Damaged mitochondria could serve as a source of oxidative radicals involved in tenofovir induced kidney damage (Ramamoorthy et al., 2012). Krambovis and colleagues also reported that tenofovir induced oxidative stress in the kidneys may be due to the overproduction of reactive oxygen species as well as the depletion of cellular antioxidant system (Krambovitis et al., 2005). Nitroso-oxidative stress and NFkB activation was also reported to contribute to TDF induced renal damage in rats. Available evidence demonstrates that certain HIV PIs could induce endothelial dysfunction, including a decrease in endothelium-dependent vasorelaxation, inhibition of nitric oxide synthase system, increase in oxidative stress and induction of mitogen-activated protein kinases (Wang et al., 2007). Also several recent reports have suggested that HIV-protease inhibitors are associated with metabolic and/or cardiovascular toxicities. Endothelial dysfunction is an initiating event in atherogenesis and may contribute to HIVassociated atherosclerosis. In vitro treatment of human umbilical vein endothelial cells with antiretroviral showed that endothelial mitochondrial dysfunction is significantly associated with increase production of oxidative radicals (Jiang et al., 2007). Similar observation was reported when human endothelial cell culture was exposed to saquinavir. Concentrationdependent increase in cell death, mainly via apoptosis which is attributed to increased intracellular oxidant production which was abrogated by incubation with the antioxidant $\mathrm{N}$-acetylcysteine was observed (Baliga et al., 2004). Mondal et al. (2004), reported that oxidative stress can disrupt endothelial homeostasis by dysregulating the balance between pro- and antiatherogenic factors. They added credence to their reports by showing that chronic HAART exposure increases oxidative stress in endothelial cells and induces mononuclear cell recruitment which may eventually precipitate cardiovascular diseases observed in HIV-1 positive individuals on antiretroviral therapy
(Shankar et al., 2005; Shlay et al., 2007; Valko et al., 2006). Other studies have shown that protease inhibitors induced endoplasmic reticulum stress in many cell types including hepatocytes, macrophages and intestinal epithelial cells probably via the generation of oxidative radicals (Zhou et al., 2006; Wu et al., 2010; Djedaini et al., 2009; Cho et al., 2009; Pyrko et al., 2007).

\subsection{Antiretroviral Induced Oxidative Stress in Humans}

Quite a number of studies have reported impaired antoxidants and antioxidants enzyme in HIV patients treated with highly active antiretroviral therapy which could be correlated with their toxicological effects. It has been proposed that toxicological effects of antiretroviral drugs like myopathy, cardiomyopathy, anaemia, hyperlactataemia/lactic acidosis, pancreatitis, polyneuritis and lipodystrophy could be mediated through mitochondrial toxicity. Several studies relating oxidative stress with highly active antiretroviral therapy through mitochondrial dysfunction were reported by some scholars (Masia et al., 2007; Mandas et al., 2009; Gil et al., 2010; Sundaram et al., 2008). Mitochondria DNA inhibition may cause energy deprivation and increase reactive oxygen species formation. Oxidative stress-mediated cell damage may occur via reactive oxygen species production induced by some antiretroviral drugs (Vassimon et al., 2010). Ngondi et al. (2006) assessed the effects of different highly active antiretroviral combinations on oxidative stress parameters and found an increase in lipid oxidation and decrease in antioxidants. Hulgan et al. (2003) quantified plasma F2 isoprostanes as an oxidative stress index that could be induced by antiretrovirals. They evaluated different strategies and found that this index increased in one hundred and twenthy HIV positive patients on highly active antiretroviral therapy.

Treatment of HIV patients with highly active antiretroviral therapy containing (Zidovudine, Lamivudine Nevirapine) and (Zidovudine, Lamivudine, Efavirenz) was reported to decrease selenium levels in some patients and increased production of free radicals (Atiba et al., 2012). This is also supported by Masia et al. (2007) who reported that antiretroviral therapy also plays a role in oxidative damage to DNA and membrane polyunsaturated fatty acid, which could lead to the generation of more free radicals potentiating cellular damage (Masia et al., 2007). Antiretroviral combination 
therapies were reported to increased protein oxidation as well as the level of oxidative stress already present in HIV infection (Ngondi et al., 2006). Researchers have shown that exposure to protease inhibitors especially nelfinavir, suppresses glucose mediated insulin secretion in pancreatic b-cells. This effect could be mediated through increased oxidative stress as reported (Chandra et al., 2009). Recent evidence showed that adjunct therapy with antioxidants may ameliorate these deleterious effects of highly active antiretroviral regimens containing protease inhibitors (Ben-Romano et al., 2006).

Gil et al. (2010) evaluated the effect of two highly active antiretroviral therapy (Zidovudine, Lamivudine, Indinavir) and (Stavudine, Lamivudine, Nevirapine) combinations on redox indicators. They reported increase in oxidative stress which occurred additionally to persistent redox imbalance associated with HIV-1 infection. Mandas et al. (2009) assessed serum oxidant and antioxidant levels in HIV-1 infected patients on highly active antiretroviral therapy and made comparism with those untreated HIV-1 seropositive and HIV-1 seronegative individuals. Serum oxidant levels were significantly higher in the HIV-1 treated group with respect to the untreated and control groups. In addition, decrease in serum total antioxidant status was observed in HIV-1 treated individuals. Highly active antiretroviral therapy may increase oxidative radicals in circulation, possibly by producing more oxidized metabolites derived from the interaction between ROS and infected-cell biomolecules. This is supported by several biochemical mechanisms, such as mitochondrial interference, following highly active antiretroviral therapy (Lewis, 2003; Cossarizza and Moyle, 2004; Day and Lewis, 2004). Clinical trials have been conducted to determine the effect of antioxidant supplements in HIV infection. Results indicated beneficial effects of antioxidants supplementation against the progression of HIV infection as reported by (Milazzo et al., 2010; Yousefi et al., 2011; Hurwitz et al., 2007).

\section{CONCLUSION}

Antiretroviral drugs are associated with toxicological effects and induction of oxidative stress via the generation of oxidative radicals, decrease antioxidants and antioxidant enzymes but more studies are required to correlate antiretroviral toxicities with oxidative stress.

\section{REFERENCES}

Aberg, J.A., J.E. Kaplan and H. Libman, 2009. Primary care guidelines for the management of persons infected with human immunodeficiency virus: Update by the HIV medicine association of the infectious diseases society of america. Clin. Infect. Dis., 49: 651-681. DOI: 10.1086/605292

Abrescia, P. and P. Golino, 2005. Free radicals and antioxidants in cardiovascular diseases. Expert Rev. Cardiovasc Ther., 3: 159-171. DOI: 10.1586/14779072.3.1.159

Adjene, J.O., J.A. Avbunudiogba and P.S. Igbigbi, 2011. Oxidative stress induced by chronic administration of efavirenz on the intracranial visual relay centers of adult wistar rats. Biol. Med., 3: 16-24.

Apostolova, N., L.J. Gomez-Sucerquia, A. Moran, A. Alvarez and A Blas-Garcia at al., 2010. Enhanced oxidative stress and increased mitochondrial mass during Efavirenz-induced apoptosis in human hepatic cells. Br. J. Pharmacol., 160: 2069-2084. DOI: $10.1111 / \mathrm{j} .1476-5381.2010 .00866 . \mathrm{x}$

Atiba, A.S., D.P. Oparinde, A.K. Jimoh, O.A. Babatunde and A. Adelekan et al., 2012. Oxidative stress and serum selenium in HIV patients on different antiretroviral regimen. Greener J. Med. Sci., 2: 163-167.

Baliga, R.S., C. Liu, D.G. Hoyt, A.A. Chaves and J.A. Bauer, 2004. Vascular endothelial toxicity induced by HIV protease inhibitor. Cardiovasc. Toxicol., 2: 199-206. DOI: 10.1385/CT:4:2:199

Barre-Sinoussi, F., J.C. Chermann, F. Rey, M.T. Nugeyre and S. Chamaret at al., 1983. Isolation of a T-lymphotropic retrovirus from a patient at risk for Acquired Immune Deficiency Syndrome (AIDS). Science, 220: 868-871. PMID: 6189183

Ben-Romano, R., A. Rudich, S. Etzion, R. Potashnik and E. Potashnik et al., 2006. Nelfinavir induces adipocyte insulin resistance through the induction of oxidative stress: Differential protective effect of antioxidant agents. Antivir. Ther., 11: 1051-1060. PMID: 17302375

Bera, E., D. Naidoo and M. Williams, 2012. Maternal deaths following nevirapine-based antiretroviral therapy. South Afr. J. HIV Med., 13: 196-197. DOI: 10.7196/sajhivmed.869

Chandra, S., D. Mondal and K.C. Agrawal, 2009. HIV-1 protease inhibitor induced oxidative stress suppresses glucose stimulated insulin release: Protection with thymoquinone, experim. Biol. Med., 234: 442-453. DOI: 10.3181/0811-RM-317 
Cho, H.Y., S. Thomas, E.B. Golden, K.J. Gaffney and F.M. Hofman et al., 2009. Enhanced killing of chemo-resistant breast cancer cells via controlled aggravation of ER stress. Cancer Lett., 282: 8797. DOI: 10.1016/j.canlet.2009.03.007, PMID: 19345476

Cooper, D., J. Heera, J. Goodrich, M. Tawadrous and M. Saag et al., 2010. Maraviroc versus efavirenz, both in combination with zidovudine-lamivudine, for the treatment of antiretroviral-naïve subjects with CCR5-tropic HIV-1 infection. J. Infec. Dis., 201: 803-813. DOI: 10.1086/650697

Cossarizza, A. and G. Moyle, 2004. Antiretroviral nucleoside and nucleotide analogues and mitochondria. AIDS, 18: 137-151. PMID: 15075530

Day, B.J. and W. Lewis, 2004. Oxidative stress in NRTIinduced toxicity: Evidence from clinical experience and experiments in vitro and in vivo. Cardiovascular Toxicol., 4: 207-216. PMID: 15470269

De Clercq, E., 2009. Anti-HIV drugs: 25 compounds approved within 25 years after the discovery of HIV. Int. J. Antimicrob. Agents, 33: 307-320. PMID: 19108994

Djedaini, M., P. Peraldi, M.D. Drici, C. Darini and P. Saint-Marc et al., 2009. Lopinavir co-induces insulin resistance and ER stress in human adipocytes. Biochem. Biophy. Res. Commun., 386: 96-100. DOI: 10.1016/j.bbrc.2009.05.14

Domingo, P. and F. Lozano, 2011. Management of antiretroviral drug toxicity. Enfermedades Infecciosas Mic. Clin., 29: 535-544. DOI: 10.1097/01.COH.0000239856

Elias, A. and N. Brambaifa, 2013. Concentration-effect, incidence and mechanism of nevirapine hepatotoxicity. Am. J. Pharmacol. Toxicol., 8: 2030. DOI: 10.3844/ajptsp.2013.20.30

Elias, A., O. Deo, O.B.P. Geoffrey and D.A. Enimeya, 2013. Nevirapine hepatotoxicity: Implications of risk factors. Am. J. Pharm. Toxicol., 8: 51-63. DOI: 10.3844/ajptsp.2013.51.63

Elsayed, R.K. and D.J. Caldwell, 2010. Etravirine a novel nonnucleoside reverse transcriptase inhibitor for managing human immunodeficiency virus infection. Am. J. Health. Syst. Pharm., 67: 193-205. DOI: 10.2146/ajhp080559

Emery, S. and A. Winston, 2009. Raltegravir: A new choice in HIV and new chance for research. Lancet, 374: 764-766. DOI: 10.1016/S0140-6736, PMID: 19647865
Eron, J.J., B. Young, A. Cooper, M. Youle and P. Sklar et al., 2010. Switch to a raltegravir-based regimen versus continuation of a lopinavir-ritonavir-based regimen in stable $\mathrm{HIV}$-infected patients with suppressed viraemia (SWITCHMRK 1 and 2): Two multicentre, double-blind, randomised controlled trials. Lancet, 375: 396-407. DOI: 10.1016/S01406736(09)62041-9

Ferraresi, R., L. Troiano, E. Roat, E. Nemes and E. Lugli at al., 2006. Protective effect of acetyl-L-carnitine against oxidative stress induced by antiretroviral drugs. FEBS Lett., 580: 6612-6616. DOI: 10.1016/j.febslet.2006.11.016

Gallant, J.E. and S. Deresinski, 2003. Tenofovir disoproxil fumarate. Clin. Infec. Dis., 37: 944-950. DOI: $10.1086 / 378068$

Gerschenson, M. and K. Brinkman, 2004. Mitochondrial dysfunction in AIDS and its treatment. Mitochondrion, 4: 763-777. DOI: $10.1086 / 605292$

Gil, L., A. Tarinas, D. Hernadez, B.V. Riveron and D. Perez et al., 2010. WITHDRAWN: Altered oxidative stress indexes related to disease progression marker in human immunodeficiency virus infected patients with antiretroviral therapy. Biomed. Pharmacother. PMID: 20951539

Glesby, D.R., 2002. Overview of mitochondrial toxicity of nucleoside reverse transcriptase inhibitors.

Guo, Y. and H.B. Fung, 2004. Fatal lactic acidosis associated with coadministration of didanosine and tenofovir disoproxil fumarate. Pharmacotherapy, 24: 1089-1094. PMID: 15338857

Hassan, A., A.A. Babadoko, A.I. Mamman and S.A. Ahmed, 2009. Zidovudine induced pure red cell aplasia: A case report. Niger J. Med.,18: 332-333. PMID: 20120657

Havlir, D.V., 2008. HIV integrase inhibitors. out of the pipeline and into the clinic. N. Engl. J. Med., 359: 416-418. DOI: 10.1056/NEJMe0804289

Hill, A., W. Sawyer and B. Gazzard, 2009. Effects of first-line use of nucleoside analogues: Efavirenz and ritonavir-boosted protease inhibitors on lipid levels. HIV Clin. Trials., 10: L1-S12. PMID: 19362991

Huang, J., L. Meixner, S. Fernandez and J.A. McCutchan, 2009. A double-blinded, randomized controlled trial of zoledronate therapy for HIVassociated osteopenia and osteoporosis. AIDS, 23: 51-57. DOI: 0.1097/QAD.0b013e32831c8adc 
Hughes, P.J., E. Cretton-Scott, A. Teague and T.M. Wensel, 2011. Protease inhibitors for patients with HIV-1 infection: A comparative overview. P T., 36: 332-345. PMID: 21785550

Hulgan, T., J.D. Morrow, R.T. D’Aquila, S. Raffanti and M. Morgan et al., 2003. Oxidant stress is increasing during treatment of human immunodeficiency virus infection. Clin. Infect. Dis., 37: 1711-1717. PMID: 14689356

Hurwitz, B.E., J.R. Klaus, M.M. Llabre, A. Gonzalez and P.J. Lawrence et al., 2007. Suppression of human immunodeficiency virus type 1 viral load with selenium supplementation. Arch. Intern. Med., 167: 148-154. DOI: 10.1001/archinternmed.2012.2706

James, C.L.P., P. Lee and M. Sweet, 2012. Rilpivirine: A second-generation nonnucleoside reverse transcriptase inhibitor. Am. J. Health-Syst. Pharm., 69: 857-861. DOI: 10.2146/ajhp110395

Jena, A., R.K. Sachdeva, A. Sharma and A. Wanchu, 2009. Adverse drug reactions to nonnucleoside reverse transcriptase inhibitor-based antiretroviral regimen: A 24-week prospective Study. J. Int. Assoc. Providers of AIDS Care, 8: 318-322. DOI: 10.1177/1545109709343967

Jiang, B., V.Y. Hebert A.R. Khandelwal K.Y. Stokes and T.R. Dugas, 2009. HIV-1 antiretrovirals induce oxidant injury and increase intimamedia thickness in an atherogenic mouse model. Toxicol. Lett., 187: 164-171. DOI: 10.1016/j.toxlet.2009.02.017

Jiang, B., V.Y. Hebert, Y. Li, J.M. Mathis and J.S. Alexander et al., 2007. HIV antiretroviral drug combination induces endothelial mitochondrial dysfunction and reactive oxygen species production, but not apoptosis. Toxicol. Applied Pharmacol., 224: 60-71. DOI: 1016/j.taap.2007.06.010

Kaferle, J. and C.E. Strzoda, 2009. Evaluation of macrocytosis. Am. Fam. Phys., 1: 203-208.

Kashou, A.H. and A. Agarwal, 2011. Oxidants and antioxidants in the pathogenesis of HIV/AIDS. Open Reproductive Sci. J., 3: 154-161. DOI: 10.2174/1874255601103010154

Katlama, L.P., R. Haubrich and J. Lalezaric, 2009. Efficacy and safety of etravirine in treatment experienced, HIV-1 patients: Pooled 48-week analysis of two randomized, controlled trials. AIDS, 23: 2289-2300. DOI: 10.1097/QAD.0b013e3283316a5e
Kohler, J.J., S.H. Hosseini, A. Hoying-Brandt, E. Green and D.M. Johnson et al., 2009. Tenofovir renal toxicity targets mitochondria of renal proximal tubules. Laboratory Investigat., 89: 513-519. DOI: 10.1038/labinvest.2009.14

Krambovitis, E., F. Porichis and D.A. Spandidos, 2005. HIV entry inhibitors: A new generation of antiretroviral drugs. Acta Pharmacol. Sin., 26: 116573. DOI: $10.1111 /$ j.1745-7254.2005.00193.x

Lauderback, C.M., J. Drake, D. Zhou, J.M. Hackett and A. Castegna at al., 2003. Derivatives of xanthic acid are novel antioxidants: Application to synaptosomes. Free Radic. Res., 37: 355-365. DOI: 10.1080/1071576021000040664

Lennox, J.L., E. DeJesus and A.A. Lazzarin, 2009. STARTMRK investigators. Safety and efficacy of raltegravir-based versus efavirenz-based combination therapy in treatment-naive patients with HIV-1 infection: A multicentre, double-blind randomised controlled trial. Lancet, 374: 796-806. DOI: 10.1016/S0140-6736(09)60918-1

Lewis, W., 2003. Mitochondrial dysfunction and nucleoside reverse transcriptase inhibitor therapy: Experimental clarifications and persistent clinical questions. Anti. Res., 58: 189-197. PMID: 12767466

Lundgren, J., P. Reiss and S. Worm, 2009. Risk of myocardial infarction with exposure to specific ARV from the PI, NNRTI and NRTI drug classes: the D:A:D study. Proceedings of the 16th Conference on Retroviruses and Opportunistic Infections, Montreal, Feb. 8-11, Alexandria, VA, USA., pp: 318-330. DOI: 10.1086/649897

Madruga, J.V., P. Cahn, B. Grinztejn, R. Haubrich and J. Lalezari et al., 2007. Efficacy and safety of TMC125 (etravirine) in treatment-experienced HIV1-infected patients in DUET-1: 24-week results from a randomised, double-blind, placebo-controlled trial. Lancet, 370: 29-38. PMID: 17617271

Manda, K.R.A.B., W.A. Banks and N. Ercal, 2011. Highly active antiretroviral therapy drug combination induces oxidative stress and mitochondrial dysfunction in immortalized human blood-brain barrier endothelial cells. Free Radical Biol. Med., 50: 801-810. DOI: 1016/j.freeradbiomed.2010.12.029

Mandas, A., E.L. Lorio, M.G. Congiu, C. Balestrieri and A. Mereu et al., 2009. Oxidative imbalance in HIV1 infected patients treated with antiretroviral therapy. J. Biomed. Biotechnol., 18: 67-79. DOI: $10.1155 / 2009 / 749575$ 
Masia, M., S. Padilla, E. Bernal, M.V. Almenar and J. Molina et al., 2007. Influence of antiretroviral therapy on oxidative stress and cardiovascular risk: A prospective cross-sectional study in HIV-infected patients. Clin. Therap., 29: 448-455. DOI: 10.1016/j.clinthera.2007.07.025

Meanwell, N.A. and J.F. Kadow, 2007. Maraviroc, a chemokine CCR5 receptor antagonist for the treatment of HIV infection and AIDS. Curr. Opin. Invest. Drugs, 8: 669-681. PMID: 17668369

Milazzo, L., B. Menzaghi, I. Caramma, M. Nasi and O. Sangaletti et al., 2010. Effect of antioxidants on mitochondrial function in HIV-1-related lipoatrophy: A pilot study. AIDS, 26: 1207-1214. DOI: 10.1089/aid.2010.0024

Mitsuya, H., K.J. Weinhold, P.A. Furman, M.HS. Clair and S.N. Lehrman et al., 1985. 3'-Azido-3'deoxythymidine (BW A509U): An antiviral agent that inhibits the infectivity and cytopathic effect of human T-lymphotropic virus type III/lymphadenopathy-associated virus in vitro. Proc. Natl. Acad. Sci. USA., 82: 7096-7100.

Mondal, D., L. Pradhan, M. Ali and K.C. Agrawal, 2004. HAART drugs induce oxidative stress in human endothelial cells and increase endothelial recruitment of mononuclear cells. Cardiovasc. Toxicol., 4: 287-302. DOI: 10.1385/CT:4:3:287

Ngondi, J.L., J. Oben, D.M. Forkah, L.H. Etame and D. Mbanya, 2006. The effect of different combination therapies on oxidative stress markers in hiv infected patients in cameroon. AIDS Res. Therap., 3: 19-26. DOI: $10.1186 / 1742-6405-3-19$

Noor, M.A., 2007. The role of protease inhibitors in the pathogenesis of HIV-associated insulin resistance: Cellular mechanisms and clinical implications. Curr. HIV/AIDS Rep., 4: 126-134. DOI: 10.1007/s11904007-0019-4

Opii, W.O., R. Sultana, H.M. Abdul, M. Ansari and A. Nath at al., 2007. Oxidative stress and toxicity induced by the Nucleoside Reverse Transcriptase Inhibitor (NRTI)-2: 3-dideoxycytidine (ddC): Relevance to HIV dementia. Exp. Neurol., 204: 2938. DOI: 1016/j.expneurol.2006.09.010

Pallela, F., K. Delaney, A. Moorman, M. Loveless and J. Fuhrer et al., 1998. Declining morbidity and mortality among patients with advanced human immunodeficiency virus infection. New Engl. J. Med., 338: 853-860. DOI: 10.1056/NEJM199803263381301
Pocernich, C.B., R. Sultana, H. Mohmmad-Abdul, A. Nath and A. Butterfield, 2005. HIV-dementia, Tatinduced oxidative stress and antioxidant therapeutic considerations. Brain Res. Rev., 50: 14-26. ISBN 15890409

Popovic, M., P.S. Sarin, M. Robert-Gurroff, V.S. Kalyanaraman and D. Mann at al., 1983. Isolation and transmission of human retrovirus (human Tcell leukemia virus). Sci., 219: 856-859. PMID: 6600519

Pyrko, P., A. Kardosh, W. Wang, W. Xiong and A.H. Schonthal et al., 2007. HIV-1 protease inhibitors nelfinavir and atazanavir induce malignant glioma death by triggering endoplasmic reticulum stress. Cancer Res., 67: 10920-10928. DOI: 10.1158/00085472.CAN-07-0796

Ramamoorthy, H., B. Issac and P. Abraham, 2012. Evidence for the roles of oxidative stress, nitrosative stress and $\mathrm{Nf}-\mathrm{Kb}$ activation in Tenofovir Disoproxil Fumarate (TDF) induced renal damage in rats. BMC Infect. Dis., 12: 1-6. DOI: 10.1186/1471-2334-12S1-P6

Ravichandran, S., R. Veerasamy S. Raman and P.N. Krishnan and R.K. Agrawal, 2008. A overview on Hiv-1 reverse transcriptase inhibitors. Digest J. Nanomaterials Biostruct., 3: 171-187.

Reust, C.E., 2011. Common adverse effects of antiretroviral therapy for HIV disease. Am. Fam. Phys., 83: 1443-1451. PMID: 21671545

Rivero, A. and A. Jose, 2007. Mira2 and Juan A. Pineda3, Liver toxicity induced by non-nucleoside reverse transcriptase inhibitors. J. Antimicrob. Chemother., 59: 342-346. DOI: $10.1093 / \mathrm{jac} / \mathrm{dkl} 524$

Saitoh, A.T.F., C. Alvero, C.V. Fletcher and S.A. Spector, 2007. Impact of nucleoside reverse transcriptase inhibitors on mitochondria in human immunodeficiency virus type 1-infected children receiving highly active antiretroviral therapy. Antimicrob. Agents Chemother., 51: 4236-4242. PMID: 17893156

Schafer, J.J., S. Ravi, E.V. Rowland S. Ravi and N. Leon, 2011. The expanding class of non-nucleoside reverse transcriptase inhibitors for the treatment of HIV-1 infection. P T., 36: 346-364.

Schneider, C.D. and A.R. De Oliveira, 2004. Oxygen free radicals and exercise: Mechanisms of synthesis and adaptation to the physical training. Rev. Bras. Med. Esporte, 10: 314-318. DOI: 10.1590/S151786922004000400008 
Shankar, S.S., M.P. Dube, J.C. Gorski, J.E. Klaunig and H.O. Steinberg, 2005. Indinavir impairs endothelial function in healthy HIV-negative men. Am. Heart J., 150: 933-933. PMID: 16290967

Shlay, J.C., G. Bartsch, G. Peng, J. Wang and C. Grunfeld et al., 2007. Long-term body composition and metabolic changes in antiretroviral naive persons randomized to protease inhibitor-, nonnucleoside reverse transcriptase inhibitor-, or protease inhibitor plus nonnucleoside reverse transcriptase inhibitor-based strategy. J. Acquir. Immun. Defic. Syndr., 44: 506-517. DOI: 1097/QAI.0b013e31804216cf

Sultana, R., S. Newman, H. Mohmmad-Abdul, J.N. Keller and D.A. Butterfield, 2004. Protective effect of the xanthate, D609, on Alzheimer's amyloid betapeptide (1-42)-induced oxidative stress in primary neuronal cells. Free. Radic. Res., 38: 449-458. DOI: 10.1080/1071576021000040664

Sundaram, M., S. Saghayam, B. Priya, K.K. Venkatesh and P. Balakrishnan, 2008. Changes in antioxidant profile among HIV-infected individuals on generic highly active antiretroviral therapy in Southern India. Int. J. Infect. Dis., 12: 61-66. DOI: 10.1016/j.ijid.2008.04.004

Suneeta, S., R. Churchill and Duncan and Y. Gilleece, 2008. Abacavir-induced hepatotoxicity: A report of two cases. AIDS, 22: 2557-2562. DOI: 1097/QAD.0b013e32831c8af4

Tebas, P., 2008. Insulin resistance and diabetes mellitus associated with antiretroviral use in HIV-infected patients: Pathogenesis, prevention and treatment options. J. Acquir. Immun. Defic. Syndr., 49: S8692. DOI: 1097/QAI.0b013e31818651e6

Terry, L., E. Sprinz, R. Stein, N.B. Medeiros and J. Oliveira et al., 2006. Exercise training in HIV-1infected individuals with dyslipidemia and lipodystrophy. Med. Sci. Sports. Exerc., 38: 411 417. DOI: 10.1249/01.mss.0000191347.73848.80

Valko, M., C.J. Rhodes, J. Moncol, M. Izakovic and M. Mazur, 2006. Free radicals, metals and antioxidants in oxidative stress-induced cancer. Chem. Biol. Interact., 160: 1-40. DOI: 1016/j.cbi.2005.12.009

Valle, L.G.D., R.G. Hernandez and J.P. Avila, 2013. Oxidative stress associated to disease progression and toxicity during antiretroviral therapy in human immunodeficiency virus infection. J. Virol. Microbiol. DOI: 10.5171/2013.279685
Van Huyen, J., A. Landau, C. Piketty and M.F. Belair, 2003. Toxic effects of nucleoside reverse transcriptase inhibitors on the liver. Am. J. Clin. Pathol., 119: 546-555. DOI: 10.1309/8B8BJ6AP5KGV7C1H

Vassimon, H.S., R. Deminice, A.A. Machado, J.P. Monteiro and A. Jordao, 2010. The association of lipodystrophy and oxidative stress biomarkers in HIV-infected men. Curr. HIV Res., 8: 364-369. DOI: $10.2174 / 157016210791330347$

Wang, X., H. Chai, Q. Yao and C. Chen, 2007. Molecular mechanisms of HIV protease inhibitorinduced endothelial dysfunction. J. Acquired Immune Deficiency Syndr., 44: 493-499. DOI: 10.1097/QAI.0b013e3180322542

Wensing, A., N. van Maarseveen and M. Nijhuis, 2010. Fifteen years of HIV protease inhibitors: Raising the barrier to resistance. Antiviral Res., 85: 59-74. DOI: 1016/j.antiviral.2009.10.003

$\mathrm{Wu}, \mathrm{X}$., L. Sun, W. Zha, E. Studer and E. Gurley et al., 2010. HIV protease inhibitors induce endoplasmic reticulum stress and disrupt barrier integrity in intestinal epithelial cells. Gastroenterology, 138: 197-209. DOI: $10.1053 /$ j.gastro.2009.08.054

Young, B., K. Buchacz, A. Moorman, K.C. Wood and J.T. Brooks, 2009. Renal function in patients with preexisting renal disease receiving tenofovircontaining highly active antiretroviral therapy in the HIV outpatient study. AIDS Patient Care STDS, 23: 589-592. DOI: 10.1089/apc.2008.0232

Yousefi, S., A. Hadadi, A. Ostovar, B.E. Noor and M. Rasoolinejad et al., 2011. Protocol of determining the effect of selenium supplementation on CD4 + T lymphocyte count in HIV/AIDS patients: A randomized double blind placebo controlled trial. Adv. Infect. Dis., 1: 20-26. DOI: 10.4236/aid.2011.12003

Zhou, H., E.C. Gurley, S. Jarujaron, H. Ding and Y. Fang et al., 2006. HIV protease inhibitors activate the unfolded protein response and disrupt lipid metabolism in primary hepatocytes. Am. J. Physiol. Gastrointestinal Liver Physiol., 291: 1071-80. PMID: 16861219 